01,02

\title{
Микроволновой фотонный детектор
}

\author{
(C) E.B. Ильичев \\ Leibniz Institute of Photonic Technology, \\ Jena, Germany \\ Новосибирский государственный технический университет, \\ Новосибирск, Россия \\ E-mail: evgeni.ilichev@leibniz-ipht.de
}

Обсуждается возможность реализации однофотонного детектора в микроволновом диапазоне. Показано, что для этих целей можно использовать нешунтированный джозефсоновский переход, который переключается из сверхпроводящего состояния в состояние с конечным напряжением при наличии внешнего сигнала. Чувствительность такого детектора определяется распределением переключений при отсутствии внешнего сигнала. В работе продемонстрирован джозефсоновский переход с шумовой температуре ниже $60 \mathrm{mK}$ при номинальной внешней температуре $10 \mathrm{mK}$. Кроме того предложен, рассчитан и изготовлен конкретный детектор для измерения внешних СВЧ сигналов. Приводятся первые экспериментальные результаты.

Работа выполнена при финансовой поддержке Российского научного фонда в рамках проекта № 16-19-10069.

\section{1. Введение}

В настоящее время обсуждается несколько стратегий реализации квантового компьютера. Одно из направлений нацелено на реализацию адиабатического квантового компьютера $[1,2]$, иногда называемого квантовым симулятором. Изготовление эффективных квантовых переключателей также находится в фокусе внимания исследователей (см. например [3]). Использование идей из квантовой оптики для реализации фотонного компьютера в микроволновом диапазоне также является актуальной задачей. Здесь, кроме всего прочего, было продемонстрировано сильное нелинейное взаимодействие макроволновых фотонов с системой джозефсоновских контактов, играющих роль среды, нелинейность которой описывается константой Керра. Экспериментально полученные большие величины константы Керра указывают, в принципе, на возможность создания оптических затворов в микроволновом диапазоне [4].

Однако отсутствие эффективных однофотонных детекторов для низкоэнергетических фотонов принципиально ограничивает экспериментальную реализацию идей квантовой оптики и развитие такого перспективного направления как микроволновая квантовая инженерия. Здесь многообещающими материалами для создания масштабируемых твердотельных приборов являются сверхпроводники. Во-первых, методики изготовления тонкопленочных линий передач с малыми потерями, высокодобротных резонаторов [5] и делителей мощности микроволнового сигнала $[6,7]$ хорошо известны. Вовторых, активно исследуемые в настоящее время сверхпроводниковые квантовые биты (кубиты) [8-10] могут, в принципе, играть роль двухуровневых квантовых систем для генерации и детектирования фотонов. Более того, известные элементы сверхпроводниковой электроники, использующие нелинейное поведение джозефсоновских контактов, могут быть использованы для необходимой обработки сигналов.
Необходимо отметить, что для создания масштабируемых твердотельных приборов генерации, передачи и обработки квантовых микроволновых сигналов (сигналов, содержащих малое количество фотонов) необходимы низкие температуры. Например, для минимизации вероятности термического (температура $T$ ) возбуждения квантового осциллятора (частота $\omega$ ) необходимо выполнение условия $h \omega>k_{\mathrm{B}} T$, где $h, k_{\mathrm{B}}$ - постоянные Планка и Больцмана, соответственно. Отсюда следует, что частота $21 \mathrm{GHz}$ соответствует температуре $1 \mathrm{~K}$. Таким образом, использование сверхпроводящих материалов в микроволновой квантовой инженерии, где основным нелинейным элементов является джозефсоновский контакт, представляется вполне естественным.

\section{2. Джозефсоновский контакт как детектор на низких частотах}

Типичная вольтамперная характеристика джозефсоновского контакта с ниобиевыми электродами изображена на рис. 1. При увеличении приложенного тока контакт остается в сверхпроводящем состоянии до достижения значения критического тока $I_{C}$. В области $I_{C}$ происходит скачок в состояние с конечным напряжением, которое для туннельных переходов на основе ниобия составляет примерно $3 \mathrm{mV}$. Этот сигнал легко детектируется стандартными приборами.

Таким образом, идея детектора заключается в следующем: приложить к контакту ток (называемый током смещения $\left.I_{B}\right)$, близкий к $I_{C}$ так что напряжение на контакте равно 0. Приходящий электромагнитный сигнал эффективно увеличивает ток смещения, вызывая переход в состояние с конечным напряжением, что и служит выходным сигналом.

Для улучшения чувствительности такого детектора необходимо изучить в деталях механизмы переключе- 


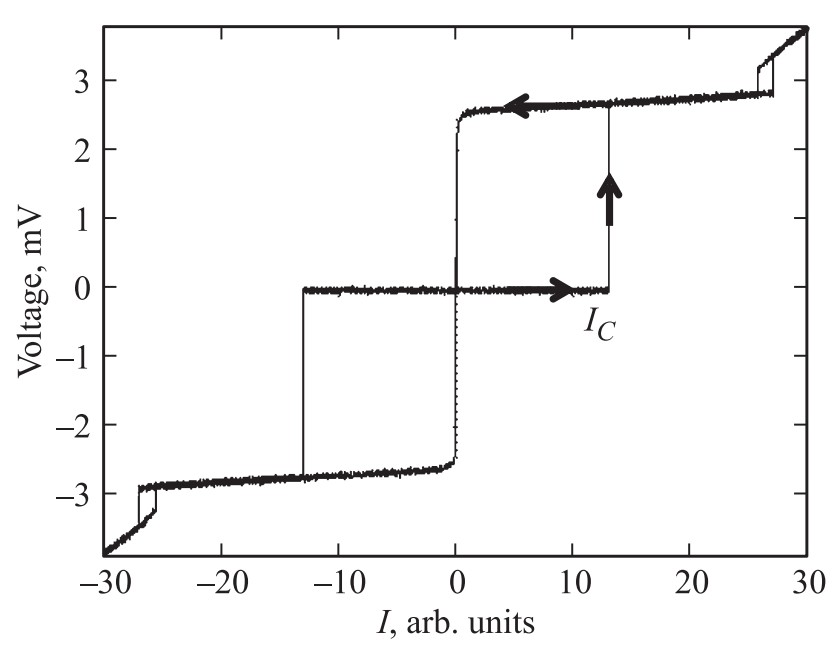

Рис. 1. Пример вольт-амперной характеристики туннельного нешунтированного контакта на основе ниобия, для которого напряжение после скачка составляет $\approx 3 \mathrm{mV}$. Стрелками указано направление развертки тока. Ток приведен в условных единицах.

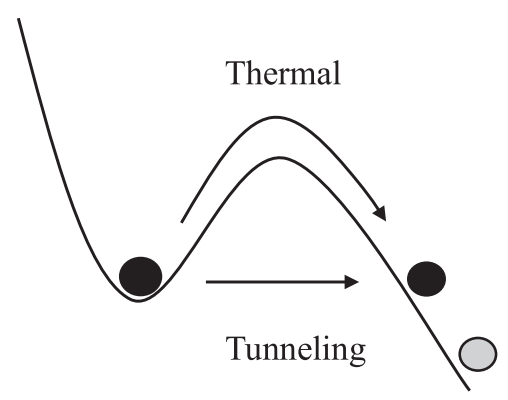

Рис. 2. Потенциал джозефсоновского туннельного нешунтированного контакта с током. Стрелками показаны возможные механизмы переключения - термический и квантовый.

ния джозефсоновского контакта. Модель здесь хорошо известна - динамика контакта описывается поведением джозефсоновской фазы. Фаза, в свою очередь, моделируется безразмерной частицей, находящейся в поле с потенциалом в форме стиральной доски (см. рис. 2). Модулирование высоты потенциального барьера или присутствие возбуждающего поля может приводить к выходу такой частицы из потенциальной ямы. Таким образом описывается появление напряжения на контакте.

В общем случае, такое переключение напряжения из нулевого состояния в конечное может быть вызвано как тепловой энергией („перескок“ через потенциальный барьер, см. рис. 2) так и макроскопическим туннелированием фазы (также см. рис. 2). При низких температурах последний режим и определяет собственный шум устройства.

Эти два процесса - термическая активация (ТА) и макроскопическое квантовое туннелирование (МКТ) имеют свои значения вероятности активации в единицу времени, $\Gamma_{T A}$ и $\Gamma_{M Q T}$ соответственно. Эти ве- личины (escape rate) определяются следующими выражениями [11]:

$$
\begin{gathered}
\Gamma_{T A}=\frac{\omega_{0}}{2 \pi} \frac{4}{\left(\sqrt{1+\frac{Q k_{\mathrm{B}} T}{1.8 \Delta U}}+1\right)^{2}} \exp \left(-\frac{\Delta U}{k_{\mathrm{B}} T}\right), \\
\Gamma_{M Q T}=\frac{\omega_{0}}{2 \pi} \sqrt{\frac{B}{2 \pi}} \exp (-B),
\end{gathered}
$$

где $\omega_{0}-$ частота осцилляции фазы в потенциальной яме, равная $\sqrt{2 \pi I_{C} / \Phi_{0} C_{K}}\left(1-I^{2}\right)^{1 / 4}, \Phi_{0}$ - квант магнитного потока, $I=I_{B} / I_{C}-$ безразмерный ток контакта, $\Delta U$ - высота потенциального барьера, определяемая джозефсоновской энергией $E_{J}, Q=\omega_{0} R_{N} C_{K}$ - добротность устройства, $R_{N}-$ сопротивление контакта, $C_{K}-$ емкость контакта, $B=\frac{\Delta U}{\hbar \omega_{0}}\left[7.2+\frac{8 A}{Q}\right], A-$ параметр, определяющий диссипацию.

Из этих выражений можно видеть, что $\Gamma_{T A}$ является функцией температуры, а $\Gamma_{M Q T}$ не зависит от температуры. Тогда очевидно, что можно вычислить температуру переключения из одного режима в другой (см. например [11])

$$
T_{\mathrm{cr}}=\frac{\hbar \omega_{0}}{2 \pi k_{\mathrm{B}}}\left[\sqrt{1+\left(\frac{1.2 A}{2 Q}\right)^{2}}-\frac{1.2 A}{2 Q}\right] .
$$

Это выражение определяет эквивалентную шумовую температуру детектора. Заметим, что для снижения эквивалентной шумовой температуры детектора можно увеличивать емкость контакта. Однако, изменение емкости будет изменять рабочий частотный диапазон детектора, и при уменьшении емкости рабочая частота также уменьшится.

Используя соображения, изложенные выше, в VTT (Финляндия) был изготовлен джозефсоновский контакт $\mathrm{Nb} / \mathrm{AlO} / \mathrm{Nb}$ со следующими параметрами: плотность критического тока $30 \mathrm{~A} / \mathrm{cm}^{2}$, емкость $C_{K}=0.33 \mathrm{pF}$, сопротивление $R_{N}=0.44 \mathrm{k} \Omega$ [11]. Образец измерялся в IPHT (Jena, Германия), в рефрижераторе растворения при номинальной температуре $10 \mathrm{mK}$. Для минимизации внешнего шума, (что является принципиальным для данной задачи!) образец, во-первых, помещался внутрь магнитного и сверхпроводящего экранов. Во-вторых, при измерении зависимости напряжения от тока использовались отфильтрованные витые пары с $R C$-фильтрами с частотой среза $10 \mathrm{kHz}$ на ступени $4 \mathrm{~K}$. Дополнительно на ступени $10 \mathrm{mK}$ располагались как $L C$-фильтры с такой же частотой среза, так и фильтры поглощения с использованием медного порошка (copper-powder filter). Электрический ток разворачивали по следующему закону: он изменялся ступенями по $0.1 \mathrm{nA} / \mathrm{ms}$ в течение $10 \mathrm{~ms}$ с временем ожидания $10 \mathrm{~ms}$ между ступенями. Экспериментально полученная ширина переключения этого автономного перехода при температуре $10 \mathrm{mK}$ составила всего $4.5 \mathrm{nA}$, и соответствующая шумовая температура составила $\approx 60 \mathrm{mK}$ [11]. 
Для сравнения оценим амплитуду тока, соответствующую одному фотону с частотой $3 \mathrm{GHz}$, которая определяется выражением $I_{A}=\sqrt{\frac{\hbar \omega}{2 \pi L_{R}}}$, где $L_{R}-$ индуктивность соответствующей части линии передачи. При использовании микроволнового четвертьволнового копланарного резонатора [12,13] соответствующее значение составляет $I_{A} \approx 20 \mathrm{nA}$. Таким образом, половины разброса переключения величиной $2.2 \mathrm{nA}$, которая определяет чувствительность данного прибора, вполне достаточно для детектирования одного фотона в СВЧ диапазоне.

Итак мы показали, что собственные флуктуации нешунтированного джозефсоновского контакта со специально выбранными параметрами невелики. Однако важно отметить, что здесь все измерения проводились на низкой частоте. Для анализа параметров возможного фотонного детектора необходима модель, описывающая взаимодействие контакта с квантованным электромагнитным полем.

\section{3. Джозефсоновский контакт как фотонный детектор}

Поскольку мы планируем работать с малым числом фотонов, необходимо квантовое описание переключения джозефсоновского перехода (схема такого переключения представлена на рис. 2.). Здесь система открытая и требуется использование достаточно громоздкого формализма матрицы плотности. Наши коллеги из Дании предложили изящный подход - использовать так называемый мнимый потенциал для описания эволюции фазы джозефсоновского перехода [14].

Как известно, временная эволюция замкнутой квантовой системы описывается динамикой вектора состояния системы $|\Psi(t)\rangle$, которая подчиняется уравнению Шредингера

$$
i h \frac{d}{d t}|\Psi(t)\rangle=\hat{H}(t)|\Psi(t)\rangle,
$$

где $\hat{H}(t)$ - оператор Гамильтона системы (здесь $i$ мнимая единица). Для гамильтониана $\hat{H}$, не зависящего от времени, это уравнение интегрируется, и решение записывается в виде $|\Psi(t)\rangle=\exp \left(-\frac{i}{h} \hat{H} t\right)|\Psi(0)\rangle$. Очевидно, что если гамильтониан содержит мнимую часть, то амплитуда $|\Psi(t)\rangle$ меняется и задача перестает быть обратимой. Вообще говоря, в этом случае $\hat{H}$ не является эрмитовым, что ставит под вопрос „законность“ данной процедуры. Однако проверка показывает, что для определенных задач такое описание адекватно [15]. В частности, динамика фазы джозефсоновского перехода хорошо описывается в рамках этого формализма [14].

Итак в потенциальной яме (до туннелирования) контакт описывается уравнением (3) с гамильтонианом

$$
\hat{H}(t)=-\frac{\hbar^{2}}{2 M} \frac{\partial^{2}}{\partial \varphi^{2}}-U(\varphi, t) .
$$

Здесь $U(\varphi, t)=U_{0}+U_{m v}$, где $U_{0}=E_{J}(\cos \varphi+I \varphi)-$ это потенциал контакта, смещенного по постоянному

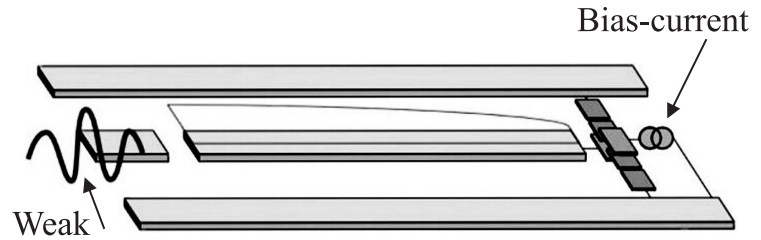

microwave

Рис. 3. Схема устройства для детектирования слабых микроволновых сигналов. После входной емкости центральный проводник резонатора закорочен смещенным джозефсоновским контактом. Две боковые сверхпроводящие полоски заземлены. Над центральным проводником схематически показана фундаментальная мода копланарного резонатора.

току $I, U_{m v}$ описывает влияние микроволнового поля на потенциал и $M$ - „эффективная масса“, равная $\left(\Phi_{0} / 2 \pi\right)^{2} C_{K}$

После акта туннелирования (вне потенциальной ямы, см. рис. 2) к потенциалу (4) добавляется мнимая часть $i V_{i m}(\varphi, t)$, вычисленная с помощью выражения для „бегущих“ состояний фазы джозефсоновского перехода, полученных в [16]. Сопротивление контакта $R$ описывается нелинейным по фазе членом (последний член в гамильтониане ниже, детали см. в [14]), и полное уравнение Шредингера записывается в виде

$$
\begin{aligned}
i h \frac{d}{d t}|\Psi(\varphi, t)\rangle=\left(-\frac{\hbar^{2}}{2 M} \frac{\partial^{2}}{\partial \varphi^{2}}-U(\varphi, t)\right)|\Psi(\varphi, t)\rangle \\
+i V_{i m}(\varphi, t)|\Psi(\varphi, t)\rangle+i(\varphi-\langle\varphi\rangle)^{2}|\Psi(\varphi, t)\rangle .
\end{aligned}
$$

Сравнение с другими моделями показывает, что данный подход имеет право на существование, в частности хорошо описывая динамику переключений контакта [14]. Однако для изготовления реального детектора фотонов этого недостаточно - связь с квантованным электромагнитным полем должна обязательно иметь место.

\section{4. Конструкция детектора}

Для связи контакта Джозефсона, смещенного по постоянному току, с измеряемым микроволновым сигналом этот джозефсоновский контакт помещается в микроволновой четвертьволновой копланарный резонатор (рис. 3). Основная идея состоит в использовнии данной структуры в качестве детектора микроволнового излучения. Захваченный резонатором фотон (фотоны) переводит контакт в состояние с конечным напряжением, что и служит выходным сигналом детектора. Копланарный резонатор можно представить как структуру, составленную из последовательно соединенных $L C$ цепей. Гамильтониан такой системы может быть записан следующим образом:

$$
H_{T}=\sum_{j} \hbar \omega_{j} a_{j}^{\dagger} a_{j}
$$

где $\omega_{j}-$ угловая частота осциллятора, $a_{j}\left(a_{j}^{\dagger}\right)-$ оператор рождения (уничтожения) фотонов в $j$-моде. 
Гамильтониан смещенного по постоянному току контакта Джозефсона был выписан в предыдущем параграфе. Таким образом, задача сводится к определению общего гамильтониана системы контакт-резонатор, который состоит из следующих частей:

$$
H=H_{T, s}+H_{J J}+H_{I},
$$

где $H_{T, s}=\hbar \omega a^{\dagger} a-$ гамильтониан одномодового режима, $H_{J J}$ - гамильтониан (4) с $U(\varphi, t)=E_{J}(\cos \varphi+I \varphi)$ и $H_{I}$ - гамильтониан, описывающий связь и взаимодействие контакта и резонатора.

Опуская детали получения полного гамильтониана системы контакт Джозефсона - копланарный резонатор, отметим, что здесь использовался стандартный формализм: сначала из уравнения движения для линии передачи был получен лагранжиан. Затем, в одномодовом приближении по стандартным правилам был получен искомый гамильтониан (подробно см. [17]).

Проведенный численный анализ полученного уравнения показал эффективность системы, достаточную для регистрации даже одиночных микроволновых фотонов. Было получено, что при оптимальной величине тока смещения $I=0.92$ квантовая эффективность системы составила 0.99 при времени детектирования $82 \mathrm{~ns}$. При этом рабочая частотная полоса детектирования составила $100 \mathrm{MHz}$ при основной частоте сигнала накачки $2.45 \mathrm{GHz}$. Предполагается, что переход к многомодовому режиму ухудшит полученные характеристики незначительно. Необходимо отметить, что контакты Джозефсона с высоким значением критического тока $I_{C}>10 \mu \mathrm{A}$ нецелесообразно использовать в структуре детектора из-за высокой величины энергии переключения, которая будет приводить к нагреву структуры и снижать чувствительность устройства.

Первые образцы были сделаны с использованием технологических процессов изготовления как контактов $\mathrm{Nb} / \mathrm{Al} / \mathrm{Nb}$ так и пленок $\mathrm{Nb}[18]$, применяемых в чистой комнате Института фотонных технологий (Jena, Германия). Тестовые измерения при номинальной температуре $10 \mathrm{mK}$ показали, что критический ток оказался немного выше запланированного и составил $13 \mu \mathrm{A}$. Щелевое напряжение контакта (напряжение после скачка) составило $2.6 \mathrm{mV}$, его сопротивление $-R_{N}=130 \Omega$ и емкость - $400 \mathrm{fF}$. Добротность резонатора, закороченного джозефсоновским контактом, составила 1100, его индуктивность - 3,5 $\mathrm{nH}$.

Несмотря на немного завышенный критический ток, образец показал свою работоспособность и переключался при подаче высокочастотного сигнала. На данный момент мы не можем однозначно определить его чувствительность, так как экспериментальный цикл еще не закончен.

Таким образом, во-первых, расчеты показывают, что предложенный детектор микроволновых фотонов может обладать чувствительностью, близкой к квантовому пределу. Во-вторых, данная структура может быть изготовлена (и первые образцы уже изготовлены!) традиционной сверхпроводниковой технологией, и измерена стандартными методами при милликельвиновых температурах.

\section{Список литературы}

[1] M.W. Johnson, M.H.S. Amin, S. Gildert, T. Lanting, F. Hamze, N. Dickson, R. Harris, A.J. Berkley, J. Johansson, P. Bunyk, E.M. Chapple, C. Enderud, J.P. Hilton, K. Karimi, E. Ladizinsky, N. Ladizinsky, T. Oh, I. Perminov, C. Rich, M.C. Thom, E. Tolkacheva, C.J.S. Truncik, S. Uchaikin, J. Wang, B. Wilson. Nature 473, 194 (2011).

[2] M. Grajcar, A. Izmalkov, E. Il'ichev. Phys. Rev. B 71, 144501 (2005).

[3] A. Fedorov, L. Steffen, M. Baur, M.P. da Silva, A. Wallraff. Nature 481, 170 (2012).

[4] M. Rehak, P. Neilinger, M. Grajcar, G. Oelsner, U. Hubner, E. Il'ichev, H.-G. Meyer. Appl. Phys. Lett. 104, 162604 (2014).

[5] P. Macha, S.H.W. van der Ploeg, G. Oelsner, E. Il'ichev, H.G. Meyer, S. Wunsch, M. Siegel. Appl. Phys. Lett. 96, 062503 (2010).

[6] P. Neilinger, G. Oelsner, M. Grajcar, Б.И. Иванов, И.Л. Новико, Е.В. Ильичев. Письма в ЖТФ 41, 7, 16 (2015); [Tech. Phys. Lett. 41, 314 (2015)].

[7] E. Hoffmann, F. Deppe, T. Niemczyk, T. Wirth, E.P. Menzel, G. Wild, H. Huebl, M. Mariantoni, T. Weiß1, A. Lukashenko, A.P. Zhuravel, A.V. Ustinov, A. Marx, R. Gross. Appl. Phys. Lett. 97, 222508 (2010).

[8] E. Il'ichev, N. Oukhanski, Th. Wagner, H.-G. Meyer, A.Yu. Smirnov, M. Grajcar, A. Izmalkov, D. Born, W. Krech, A. Zagoskin. Low Temperature Phys. 30, 620, (2004); [Fizika Nizkikh Temperatur 30, 823 (2004)].

[9] J.Q. You, F. Nori. Phys. Today 58, 11, 42 (2005).

[10] J. Clarke, F.K. Wilhelm. Nature (London) 453, 1031 (2008).

[11] G. Oelsner, L.S. Revin, E. Il'ichev, A.L. Pankratov, H.G. Meyer, L. Grönberg, J. Hassel, L.S. Kuzmin. Appl. Phys. Lett. 103, 142605 (2013).

[12] M. Jerger, S. Poletto, P. Macha, U. Hubner, A. Lukashenko, E. Il'ichev, A.V. Ustinov. Europhys. Lett. 96, 40012 (2011).

[13] M. Jerger, S. Poletto, P. Macha, U. Hubner, E. Il'ichev, A.V. Ustinov. Appl. Phys. Lett. 101, 042604 (2012).

[14] C.K. Andersen, K. Mølmer. Phys. Rev. A 87, 052119 (2013).

[15] J. Muga, J. Palao, B. Navarro, I. Egusquiza. Phys. Rep. 395, 357 (2004).

[16] G.S. Paraoanu. Phys. Rev. B 72, 134528 (2005).

[17] C.K. Andersen, G. Oelsner, E. Il'ichev, K. Mølmer. Phys. Rev. A 89, 033853 (2014).

[18] S. Anders, M. Schmelz, L. Fritzsch, R. Stolz, V. Zakosarenko, T. Schonau, H.-G. Meyer. Supercond. Sci. Technol. 22, 064012 (2009). 